\title{
Myocardial ischaemia and angina in the early post-infarction period: a comparison with patients with stable coronary artery disease
}

\author{
Bradley Marchant, Robert Stevenson, Sudhir Vaishnav, Kulasegaram Ranjadayalan, \\ Adam D Timmis
}

\begin{abstract}
Objective-To evaluate Holter and treadmill responses in patients with stable angina or recent myocardial infarction in order to compare the mechanisms of ischaemia and its symptomatic expression in these two groups.

Patients-75 patients with ischaemic ST segment depression on both a treadmill stress test and ambulatory Holter monitoring. Group A comprised 35 patients with stable angina, and group $B$ comprised 40 patients in the early period after infarction.

Setting-The coronary care unit and cardiology department of a district general hospital.
\end{abstract}

Design-A prospective, between group, comparative study.

Results-Treadmill test showed demand driven ischaemia in both groups. Although ST depression occurred at comparable rate-pressure products and workloads, it was associated with angina in $80 \%$ of group A compared with only $40 \%$ of group B $(p<0.005)$. During Holter monitoring, ST depression was associated with an attenuated increase in rate in group $A$ and almost no increase in rate in group B $(18.2 \% v 3.7 \% ; p<0.005)$, suggesting that reductions in myocardial oxygen delivery were contributing to the ischaemic episodes, particularly in group B. Ischaemic episodes were more commonly silent during Holter monitoring, particularly patients in group $B$, only two of whom experienced angina in association with ST depression. Spectral and non-spectral measures of heart rate variability were significantly reduced in group B compared with group A. in group $A$ had significantly less heart rate variability than patients who experienced angina but this difference was not dial ischaemia is usually painful and demand driven, whereas in the early period after infarction silent, supply driven ischaemia predominates. The failure of myocardial ischaemia to provoke symptoms in some patients with stable angina may be related to autonomic dysfunction affecting the sensory supply to the heart. In the early period after infarction despite clear evidence of autonomic dysfunction, other mechanisms must also be important as there was no tendency for the reduction in heart rate variability to be exaggerated in the subgroup with silent exertional ischaemia.

(Br Heart f 1993;70:438-442)

In the Framingham study, more than $10 \%$ of all myocardial infarctions were silent ${ }^{1}$ and more recent work has shown that ischaemia early after the acute attack is also commonly silent. ${ }^{2-4}$ Despite this, investigation of the pathophysiology of silent ischaemia has been largely confined to patients with stable angina and much less is known about its mechanism in acute myocardial infarction.

In stable angina, abnormalities of pain perception may account for the increased prevalence of silent ischaemia that occurs in certain clinical subgroups, particularly diabetic ${ }^{5}$ and elderly ${ }^{6}$ patients. The propensity of these patients to silent ischaemia is commonly attributed to autonomic dysfunction affecting the sensory innervation of the heart. ${ }^{8}$ Autonomic dysfunction is also common early after myocardial infarction, possibly because of destruction of neuroreceptors and neural pathways within and around the infarcted territory. ${ }^{9}$ Whether this creates zones of myocardium that are prone to developing silent ischaemia remains speculative. ${ }^{10}$

The purpose of this study was to evaluate Holter and treadmill responses in patients with stable angina and recent myocardial infarction to compare the mechanisms of ischaemia and its symptoms in these two groups. Particular attention has been given to analysis of autonomic function (as reflected by heart rate variability) and its relation to silent ischaemia.

\section{Patients and methods} STUDY DESIGN

A prospective study of ischaemia with an exercise treadmill test and ambulatory Holter monitoring for 48 hours was used to compare patients with stable angina (group A) and those after acute myocardial infarction (group B). Patients with silent exertional ischaemia seen in group $B$.

Conclusion-In stable angina, myocar-

Department of
Cardiology, London
Chest Hospital
B Marchant
R Stevenson
S Vaishnav
A D Timmis
Department of
Cardiology, Newham
General Hospital,
London
K Ranjadayalan
Correspondence to:
Dr Bradley Marchant,
Department of Cardiology,
St Bartholomew's Hospital,
West Smithfield, London
EC1A 7BE.
Accepted for publication
2 June 1993


PATIENTS WITH STABLE ANGINA (GROUP A) Consecutive patients were recruited from those undergoing an exercise treadmill test for the assessment of angina. All patients were required to have a normal resting 12 lead electrocardiogram to minimise ambiguity in interpretation of ST changes during exercise and ambulatory monitoring. Forty three such patients developed ischaemia with the exercise treadmill test (defined as $\geqslant 0.1 \mathrm{mV}$ of planar or down sloping ST segment depression) and underwent coronary angiography to confirm significant coronary disease (defined as at least one stenosis of $\geqslant 70 \%$ in one or more of the major coronary arteries). Thirty five of these patients had ischaemic episodes on 48 hour Holter monitoring (defined as $\geqslant 0.1 \mathrm{mV}$ of planar or down sloping ST segment depression) and were included in the study. Antianginal medication was withdrawn five days before the study with the exception of short acting nitrates, which were disallowed only during the study.

PATIENTS AFTER ACUTE MYOCARDIAL INFARCTION (GROUP B)

Patients were recruited from those treated with thrombolytic drugs after acute myocardial infarction. Myocardial infarction was diagnosed if two or three of the following criteria were fulfilled; typical chest pain lasting $>30$ minutes, electrocardiographic changes of $Q$ wave or non- $Q$ wave myocardial infarction, and rise in serum creatine kinase to $>400$ IU/l. Only patients with confirmed myocardial infarction and a stable hospital course were considered for inclusion, and patients with abnormalities of repolarisation caused by left bundle branch block, paced rhythms, and concurrent digoxin treatment were excluded. Two hundred and fifty six such patients underwent Holter monitoring, 102 of whom had ischaemic episodes, and 202 had exercise treadmill tests, 92 of whom showed ischaemic changes (defined as $\geqslant 0.1 \mathrm{mV}$ of planar or down sloping ST segment depression, unassociated with reciprocal ST elevation). To provide an appropriate comparison with group A, only patients with ischaemia during both tests were included. There were 40 such patients, and these comprised group $B$. Other than $\beta$-blockers, which were continued during the study, no patients were taking drugs that alter autonomic function.

\section{EXERCISE TREADMILL TEST}

This was performed according to the Bruce protocol in group $A$ and the modified Bruce protocol in group B. Exercise tests in group B were performed at a mean (SEM) of 19 (1) days after infarction (range seven to 34 days). A 12 channel electrocardiograph was monitored continuously and electrocardiograms were recorded at the onset of $1 \mathrm{~mm}$ of planar or down sloping ST segment depression, measured at $80 \mathrm{~ms}$ after the $\mathrm{J}$ point and at peak exercise. Heart rate was monitored throughout the test and blood pressure was recorded at baseline, at the onset of ischaemia, and at peak exercise. Exercise was stopped when the patient developed limiting symptoms, or, if any of the following occurred: a fall in systolic blood pressure $>10 \mathrm{~mm} \mathrm{Hg}$, significant ventricular arrhythmias, or $>5 \mathrm{~mm}$ ST depression. The occurrence of angina was noted and the workload at the onset of ischaemia and at peak exercise was recorded.

\section{AMBULATORY HOLTER MONITORING FOR 48} HOURS

All patients underwent ambulatory Holter monitoring of ST segments for 48 hours. Recordings were made in group B at 90 (5) (range 48-174) hours after the onset of infarction when the patients were mobile. Before the recording, 12 lead electrocardiograms were recorded in the sitting, standing, supine, and left and right lateral positions, to ensure that ST segments did not change with posture. Lead CMV5 and modified lead 2 were used. Recordings were made with a Marquette Series 8000 , and analysed with a Marquette Holter Acquisition Module software version $5 \cdot 8$. The time and duration of episodes of ST depression were documented. An episode was defined as ischaemia lasting for $\geqslant 1$ minute; the ST segment had to return to baseline for two minutes before a second episode could be counted. The occurrence of angina was indicated by pressing a button on the recorder and keeping a diary.

\section{HEART RATE VARIABILITY}

The first 24 hours of Holter recordings were analysed for heart rate variability with Marquette heart rate variability software. The spectral and non-spectral indices measured were: amplitude of high frequency $(0 \cdot 15-0.40$ $\mathrm{Hz})$ and low frequency $(0.04-0.15 \mathrm{~Hz})$ peaks, $S D$ of five minute mean $R R$ intervals (SDANN), root mean square of difference of successive RR intervals (rMSSD), and proportion of adjacent $R R$ intervals more than $50 \mathrm{~ms}$ different ( $\mathrm{pNN} 50$ ).

\section{STATISTICAL ANALYSIS}

All averaged results are expressed as means (SEM). Groups A and B, and exercise and Holter data were compared by the unpaired $t$ test for normally distributed variables and by the Mann-Whitney U test for other variables (duration of ischaemia and pNN50). Number of diabetic patients, sex distribution and the presence of angina on the treadmill test in groups A and B were compared with the $\chi^{2}$ test. Two sided $\mathrm{p}<0.05$ was considered significant.

The study was approved by the Newham Health District Ethics Committee, and written informed consent was given by all patients.

\section{Results}

The two groups comprised 35 patients with stable angina (group A) and 40 patients with recent myocardial infarction (group B). There were no significant differences between the groups in age (62 (2), $v 60$ (1) years), sex 
Table 1 Comparison of variables from the exercise treadmill test between groups

\begin{tabular}{|c|c|c|c|}
\hline & $\begin{array}{l}\text { Group A } \\
(n=35)\end{array}$ & $\begin{array}{l}\text { Group B } \\
(n=40)\end{array}$ & $\begin{array}{l}\text { Group B(i) } \\
(n=27)\end{array}$ \\
\hline $\begin{array}{l}\text { Heart rate (beats } / \mathrm{min}) \text { : } \\
\text { Rest } \\
\text { Onset of } 0 \cdot 1 \mathrm{mV} \mathrm{ST} \mathrm{depression} \\
\text { Peak exercise }\end{array}$ & $\begin{array}{r}81(3) \\
117(3) \\
133(3)\end{array}$ & $\begin{array}{r}83(3) \\
120(3) \\
135(3)\end{array}$ & $\begin{array}{r}89(3) \\
123(3) \\
139(4)\end{array}$ \\
\hline $\begin{array}{l}\text { Systolic blood pressure (mm Hg): } \\
\text { Onset of } 0 \cdot 1 \mathrm{mV} \mathrm{ST} \mathrm{depression} \\
\text { Peak exercise }\end{array}$ & $\begin{array}{l}149(4) \\
163(4)\end{array}$ & $\begin{array}{l}134(5)^{\star} \\
145(5)^{\star \star}\end{array}$ & $\begin{array}{l}141(7) \\
150(6)\end{array}$ \\
\hline $\begin{array}{l}\text { Rate pressure product }\left(\times 10^{3}\right) \text { : } \\
\text { Peak exercise }\end{array}$ & $21.9(0.8)$ & $19.7(0.8)$ & $20.8(0.9)$ \\
\hline $\begin{array}{l}\text { METs: } \\
\text { Onset of } 0.1 \mathrm{mV} \text { ST depression } \\
\text { Peak exercise }\end{array}$ & $\begin{array}{l}4.2(0.3) \\
6.3(0.4)\end{array}$ & $\begin{array}{l}5 \cdot 1(0 \cdot 4) \\
7 \cdot 4(0 \cdot 5)\end{array}$ & $\begin{array}{l}4 \cdot 6(0 \cdot 5) \\
7 \cdot 1(0 \cdot 6)\end{array}$ \\
\hline
\end{tabular}

${ }^{\star} \mathrm{p}<0.05$ and ${ }^{\star \star} \mathrm{p}<0.005 v$ group A. Values are means (SEM). METs, metabolic equivalents of the task. ischaemic ST depression at comparable work loads. There was no significant difference in the rate-pressure product at peak exercise. Exertional ischaemia, however, was associated with angina in $80 \%$ of patients in group A compared with only $40 \%$ of patients in group B $(p<0.005)$. This difference persisted when analysis of the responses in group $B$ was confined to those 27 patients not taking $\beta$ blockers (group $\mathrm{B}(\mathrm{i})$ ).

\section{AMBULATORY HOLTER MONITORING FOR 48 HOURS}

Relation between $S T$ depression and angina

In group $\mathrm{A}$, ischaemic ST depression was associated with angina in 23 patients $(66 \%)$, 19 of whom also had episodes of silent ischaemia. The remaining 12 patients in group A had only silent ischaemia. In group B, however, ischaemic ST depression was associated with angina in only two patients (5\%), both of whom also experienced silent ischaemia. The remaining 38 patients in group B had only silent ischaemia. Consequently, although ischaemic episodes were shorter in group A (11 (1) v 23 (4) minutes, $p<0.005$ ), the cumulative duration of painful ischaemia was much greater than in group B (993 $v 59$ minutes).

\section{Relation between ST depression and changes in heart rate \\ Immediately before episodes of ST depres- sion average heart rates were similar in groups A and B but ST depression was asso- ciated with a significantly greater increase in heart rate in group A than in group B. This difference persisted when group B data were confined to those 24 patients not taking $\beta$ - blockers (group B(i)) (table 2).}

\section{HEART RATE VARIABILITY}

Spectral and non-spectral measures of heart rate variability were significantly reduced in group B compared with group A, irrespective of treatment with $\beta$ blockers (table 3). Analysis according to the symptomatic response to treadmill exercise (table 4) showed that in group A patients who experienced no chest pain had significantly lower spectral and non-spectral measures of heart rate variability than patients who experienced agina. In group $\mathrm{B}$, on the other hand, patients with silent ischaemia on the treadmill had no reduction in heart rate variability compared with the symptomatic group, irrespective of

Table 4 Comparison of heart rate variability in different groups between patients with and without angina during the treadmill test (mean (SEM))

\begin{tabular}{|c|c|c|c|c|c|c|}
\hline & \multicolumn{2}{|l|}{ Group $A$} & \multicolumn{2}{|l|}{ Group B } & \multicolumn{2}{|l|}{ Group B(i) } \\
\hline & $\begin{array}{l}\text { Angina } \\
(n=26)\end{array}$ & $\begin{array}{l}\text { No angina } \\
(n=7)\end{array}$ & $\begin{array}{l}\text { Angina } \\
(n=13)\end{array}$ & $\begin{array}{l}\text { No angina } \\
(n=21)\end{array}$ & $\begin{array}{l}\text { Angina } \\
(n=9)\end{array}$ & $\begin{array}{l}\text { No angina } \\
(n=12)\end{array}$ \\
\hline $\begin{array}{l}\text { Spectral analysis: } \\
\text { Low frequency peak (ms) } \\
\text { High frequency peak (ms) } \\
\text { SDANN (ms) } \\
\text { rMSSD (ms) } \\
\text { pNN50 (\%) }\end{array}$ & $\begin{array}{c}25(2) \\
11(1) \\
130(7) \\
28(3) \\
7 \cdot 1(1 \cdot 8)\end{array}$ & $\begin{array}{c}17(1)^{\star \star} \\
8(1)^{\star} \\
112(10) \\
19(2)^{\star} \\
2.6(0.9)^{\star}\end{array}$ & $\begin{aligned} 13(2) \\
7(1) \\
70(5) \\
20(2) \\
2.9(0 \cdot 8)\end{aligned}$ & $\begin{array}{c}15(2) \\
8(1) \\
75(6) \\
23(2) \\
4 \cdot 8(1 \cdot 1)\end{array}$ & $\begin{array}{l}12(2) \\
6(1) \\
64(6) \\
19(2) \\
2 \cdot 9(1 \cdot 2)\end{array}$ & $\begin{array}{c}15(3) \\
8(1) \\
76(9) \\
22(3) \\
4 \cdot 8(1 \cdot 6)\end{array}$ \\
\hline
\end{tabular}

See footnote to tables 1 and 3 for abbreviations. 
Table 5 Comparison of heart rates (beats/min) before and at maximum ischaemia between the exercise treadmill test and Holter monitoring

\begin{tabular}{|c|c|c|c|c|c|c|}
\hline & \multicolumn{6}{|c|}{ Heart rate } \\
\hline & \multicolumn{2}{|c|}{ Group $A(n=35)$} & \multicolumn{2}{|c|}{ Group $B(n=40)$} & \multicolumn{2}{|c|}{ Group $B(i)(n=23)$} \\
\hline & At rest & At peak STD & At rest & At peak STD & At rest & At peak STD \\
\hline $\begin{array}{l}\text { Exercise test } \\
\text { Holter }\end{array}$ & $\begin{array}{l}81(3) \\
79(2)\end{array}$ & $\begin{array}{l}133(3) \\
92(4)^{\star \star}\end{array}$ & $\begin{array}{l}83(3) \\
77(2)\end{array}$ & $\begin{array}{l}135(3) \\
80(3)^{\star \star}\end{array}$ & $\begin{array}{l}92(4) \\
85(3)\end{array}$ & $\begin{array}{l}143(4) \\
86(3)^{\star \star}\end{array}$ \\
\hline
\end{tabular}

${ }^{\star \star} \mathrm{p}<0.005$ heart rate on Holter monitoring $v$ heart rate on exercise for a particular group. STD, ST segment depression. Other explanations as for table 1 .

treatment with $\beta$ blockers . Two recordings in group A and six recordings in group B were of inadequate quality for analysis of heart rate variability.

\section{EXERCISE TREADMILL TEST $v$. AMBULATORY} HOLTER MONITORING

The resting heart rate for both tests was similar and showed no significant differences between groups A and B (table 5). During the exercise test, ischaemic ST depression was associated with a sharp increase in heart rate in both groups but during Holter monitoring it was associated with a much smaller increase, particularly in group B. Consequently, in both groups the heart rate at peak ST depression was significantly higher during the exercise test than during Holter monitoring. This difference persisted when analysis of the responses in group B was confined to group $B(i)$.

\section{Discussion}

This study of myocardial ischaemia has provided a direct comparison between patients with stable angina and patients with recent myocardial infarction. The data point to important differences between the mechanisms of ischaemia and its symptoms in the two groups. Nevertheless, it is clear from the treadmill data that in both groups demand driven myocardial ischaemia can be provoked. Thus exercise produced similar increments in heart rate and blood pressure until the inability of oxygen supply in the diseased coronary arteries to keep pace with increasing demand led to ischaemic changes on the electrocardiogram. The ST changes occurred at almost identical average workloads and ratepressure products, confirming that during the treadmill test increased oxygen demand was the principle mechanism of ischaemia.

Periodic increments in oxygen demand may also have contributed to ischaemia during Holter monitoring, particularly in the patients with stable angina in whom ST change was associated with a significant increase in heart rate. This is unlikely, however, to be the only mechanism because the increase in heart rate was considerably lower than that which provoked ST depression during the treadmill stress test, suggesting that reductions in myocardial oxygen delivery contributed greatly to ischaemia during Holter monitoring. Our data do not permit firm conclusions about the cause of these inappropriate reductions in oxygen delivery (presumably they relate to alterations in coronary vasomotor tone) but the finding that patients with stable angina are susceptible to both demand driven and supply driven ischaemia during Holter monitoring is consistent with reports from other investigators. ${ }^{11}$

By contrast with the patients with stable angina, the patients studied early after myocardial infarction showed ST depression with almost no increase in heart rate during Holter monitoring. Clearly, these ischaemic episodes cannot be readily explained by alterations in oxygen demand, unlike those documented during the treadmill stress test. Periodic reductions in oxygen delivery, therefore, are likely to be the dominant mechanism of ischaemia in this group, a conclusion supported by the findings of Currie and Saltissi. ${ }^{12}$ These investigators showed that increments in heart rate during ischaemia on Holter monitoring in the early period after infarction (six days) were less pronounced than later recordings (38 days) when demand driven ischaemia is likely to become more prominent. Again, our data do not permit firm conclusions about the mechanism of supply driven ischaemia early after infarction, but it is possible to speculate that it relates to continuing instability of the coronary plaque in the infarct related artery after successful thrombolytic treatment, with intermittent platelet aggregation and thrombus formation leading to variable luminal obstruction and reductions in oxygen delivery. This is analogous to the mechanism that explains the increased risk of reinfarction early after thrombolytic treatment.

Ischaemic episodes were more commonly silent in the patients with recent infarction than in the patients with stable angina. It has been suggested that the propensity of patients with recent infarction to silent ischaemia reflects damage to the sensory innervation of the heart. ${ }^{13}$ Certainly the heart rate variability data in our study points to significant autonomic dysfunction in the group with recent infarction and, because myocardial sensory afferents are thought to reside in the autonomic supply, this may have contributed to the heightened susceptibility to silent ischaemia during the treadmill test and Holter monitoring. Nevertheless, this is unlikely to provide the full explanation because in the subgroup analysis (table 4) there was no evidence of exaggerated autonomic dysfunction in those group B patients with silent exertional ischaemia, suggesting that other mechanisms apart from neuropathy must also be involved in reducing ischaemic pain perception early after myocardial infarction.

Although this study casts some doubt on the hypothesis that damage to the autonomic innervation of the heart is the major mechanism of reduced ischaemic pain perception early after myocardial infarction, it provides new evidence to support a role for neuropathic mechanisms in the silent exertional ischaemia that occurs in some patients with stable angina. Thus heart rate variability was significantly reduced in those group A 
patients in whom the treadmill test provoked ischaemic ST depression unassociated with chest pain. This is analogous to findings in other groups of patients susceptible to silent ischaemia, particularly diabetic and elderly patients in whom the altered perception of angina has been associated with dysfunction of the autonomic supply to the heart. ${ }^{5714}$

Interpretation of the findings must take account of the differences between the groups. Thus the patients with stable angina were selected because of the history of angina and this no doubt accounts in part for their higher prevalence of symptomatic ischaemia. It cannot, however, account for the very low prevalence of symptomatic ischaemia in the group with recent infarction, nor for other differences in responses to the treadmill stress test and Holter monitoring that were found between the groups. A further difference is that patients in group A were selected on the basis of a normal resting electrocardiogram to optimise interpretation of ST changes. Thus no patients in this group had had a previous $Q$ wave myocardial infarction, which contrasts with group B, all of whom had recent myocardial infarction. Hence our conclusions concerning mechanisms of angina cannot necessarily be extended to patients who have had a myocardial infarction in the more distant past. As regards exercise protocols, a low level test was considered appropriate and safe in the early period after infarction and the full Bruce protocol was chosen for patients with stable angina to increase the likelihood of an ischaemic result. ${ }^{15} \mathrm{Had}$ the Bruce protocol been used in the patients with recent infarction, a higher workload might have been achieved. ${ }^{15}$ This would not have affected the main conclusions of the study. Another factor requiring consideration is that treatment with $\beta$ blockers was not withheld in patients with recent infarction. Because $\beta$ blockers can modify ischaemic and symptomatic responses during the treadmill test and Holter monitoring, a separate analysis was undertaken for the subgroup of patients not on treatment. The major differences between the groups persisted, confirming that they cannot be attributed to the effects of $\beta$ blockers.

In conclusion, myocardial ischaemia in both stable angina and recent infarction may be symptomatic or silent and may be demand driven or supply driven. This comparative study has confirmed that in stable angina painful, demand driven ischaemia is relatively more common whereas in the early period after infarction silent, supply driven ischaemia predominates. The data indicate that the failure of myocardial ischaemia to provoke symptoms in some patients with stable angina may be related to autonomic dysfunction affecting the sensory supply to the heart. In the early period after infarction, despite clear evidence of autonomic dysfunction, other mechanisms must also be important as there was no tendency for the reduction in heart rate variability to be exaggerated in the subgroup with silent exertional ischaemia.

B Marchant is funded by the British Heart Foundation

1 Margolis JR, Kannal WS, Feinleib M, Dawber TR, McNamara PM. Clinical features of unrecognised myocardial infarction-silent and symptomatic. Eighteen year follow up: the Framingham study. $A m \mathcal{F}$ Cardiol 1973;32:1-7.

2 Moczurad KW, Grodecki JK, Dubiel JP, Curylo AM Silent myocardial ischaemia in Holter monitoring and exercise stress testing after a first myocardial infarction. Eur Heart $\mathcal{~ 1 9 8 8 ; 9}$ (Suppl N):114-8.

3 Fox JP, Beattie JM, Salih MM, Davies MK, Littler WA, Murray RG. Silent ischaemia following myocardial infarction: frequency, characteristics and prognosis. Eur Heart $\mathcal{F} 1988 ; 9$ (Suppl N):108-13.

4 Ouyang P, Chandra NC, Gottlieb SO. Frequency and importance of silent myocardial ischemia identified with ambulatory electrocardiographic monitoring in the early in-hospital period after acute myocardial infarction. $\mathrm{Am}$ f Cardiol 1990;65:267-70.

5 Umachandran V, Ranjadayalan K, Ambepityia G, Marchant B, Timmis AD. The perception of angina in diabetes: relation to somatic pain threshold and autodiabetes: relation to somatic pain threshold and

6 Miller PF, Sheps DS, Bragdon EE, Herbst MC, Dalton $J \mathrm{~L}$, Hinderliter AL, et al. Aging and pain perception in ischemic heart disease. Am Heart f 1990;120:22-30.

7 Umachandran V, Ranjadayalan $\mathrm{K}$, Ambepityia G Marchant B, Kopelman PG, Timmis AD. Aging, autonomic function, and the perception of angina. Br Heart $\mathcal{f} 1991 ; 66: 15-8$.

8 Langer A, Freeman MR, Josse RG, Steiner G, Armstrong PW. Detection of silent myocardial ischemia in diabetes mellitus. Am F Cardiol 1991;67:1073-8.

9 Ewing DJ. Heart rate variability: an important new risk factor in patients following myocardial infarction. Clin factor in patients followir
Cardiol 1991;14:683-5.

10 Inoue H, Skals B, Zipes D. Effects of ischemia on cardiac afferent sympathetic and vagal reflexes in dog. $A m \mathcal{F}$ Physiol 1988;255:425-35.

11 Deedwania PC, Nelson JR. Pathophysiology of silent myocardial ischemia during daily life. Hemodynamic evaluation by simultaneous electrocardiographic and blood pressure monitoring. Circulation 1990;82: 1296-304.

12 Currie $P$, Saltissi $S$. Transient myocardial ischaemia after acute myocardial infarction. $B r$ Heart $f$ 1990;64: 299-303.

13 Yeung AC, Barry J, Selwyn AP. Silent ischemia after myocardial infarction. Prognosis, mechanism, and intervention. Circulation 1990;82:II 143-8.

14 Ambepityia G, Kopelman PG, Ingram D, Swash M, Mills PG, Timmis AD. Exertional myocardial ischemia in PG, Timmis AD. Exertional myocardial ischemia in diabetes: a quantitative analysis of anginal perceptual threshold and the influence of

15 Panza JA, Quyyumi AA, Diodati JG, Callahan TS, Epstein SE. Prediction of the frequency and duration of ambulatory myocardial ischemia in patients with stable coronary artery disease by determination of the ischemic threshold from exercise testing: importance of the exercise protocol. F Am Coll Cardiol 1991;17:657-63. 